\title{
Adsorption of aflatoxin B1, zearalenone and ochratoxin A by microorganisms isolated from Kefir grains
}

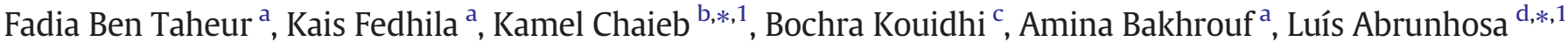 \\ a Laboratory of Analysis, Treatment and Valorization of Environmental Pollutants and Products, Faculty of Pharmacy, Monastir University, Tunisia \\ b College of Sciences, Biology Department, Yanbu el Bahr, Taibah University, Al Madinah Al Monawarah, Saudi Arabia \\ c College of Applied Medical Sciences, Medical Laboratory Department, Yanbu el Bahr, Taibah University, Al Madinah Al Monawarah, Saudi Arabia \\ d CEB - Centre of Biological Engineering, University of Minho, 4710-057 Braga, Portugal
}

\section{A R T I C L E I N F O}

\section{Article history:}

Received 2 January 2017

Received in revised form 11 March 2017

Accepted 27 March 2017

Available online 28 March 2017

\section{Keywords:}

Kefir

Adsorption

Mycotoxins

Lactobacillus kefiri

Acetobacter syzygi

Kazachstania servazzii

\begin{abstract}
A B S T R A C T
A strategy to reduce the deleterious effects of mycotoxins is to use dietary supplements that contain microorganisms that bind mycotoxins and decrease their gastrointestinal absorption. Novel strains were isolated from a Kefir culture and assessed for their mycotoxin adsorption and biotransformation ability. The most active strains were identified using DNA sequencing, and the stability of microorganism/mycotoxin complexes was evaluated using buffer solutions to simulate the $\mathrm{pH}$ conditions in the gastrointestinal tract. Our results showed that the microorganism consortium of Kefir grains adsorbed 82 to $100 \%$ of aflatoxin B1 (AFB1), zearalenone (ZEA) and ochratoxin A (OTA) when cultivated in milk. The main strains that were capable of mycotoxin adsorption were identified as Lactobacillus kefiri, Kazachstania servazzii and Acetobacter syzygii. The strain L. kefiri KFLM3 was the most active, adsorbing 80 to $100 \%$ of the studied mycotoxins when cultivated in milk. Nonetheless, the strain K. servazzii KFGY7 retained more mycotoxin after the desorption experiments $(65,69$ and $67 \%$ for AFB1, OTA and ZEA, respectively). These findings suggest that Kefir consumption may help to reduce gastrointestinal absorption of these mycotoxins and consequently reduce their toxic effects. The isolated strains may be of interest for the development of fermented dairy products for human consumption that have a new probiotic characteristic, the adsorption of mycotoxins.
\end{abstract}

(c) 2017 Elsevier B.V. All rights reserved.

\section{Introduction}

Mycotoxins are ubiquitous secondary metabolites that are produced by filamentous fungi mainly belonging to the genera Aspergillus, Penicillium and Fusarium. Mycotoxins can be found in food and animal feed around the world (Monbaliu et al., 2009). The Food and Agriculture Organization (FAO) estimated that approximately $25 \%$ of food production is contaminated with at least one mycotoxin (CAST, 1989). Humans are directly exposed to mycotoxins through consumption of contaminated plant-derived foods when agricultural commodities are colonized by mycotoxigenic fungi or indirectly by consumption of animal-derived products when livestock are fed mycotoxin-containing feed (Zain, 2011). A typical example is the carry-over of mycotoxins and their derivatives into milk. In ruminant animals, ingested mycotoxins can be metabolized by the rumen microbiota or animal organism and excreted in milk. The carry-over into milk of aflatoxin M1 (AFM1), aflatoxicol, cyclopiazonic acid, fumonisin B1 (FB1), ochratoxin A (OTA), T-2 toxin,

\footnotetext{
* Corresponding authors.

E-mail addresses: kchaieb@taibahu.edu.sa (K. Chaieb), luisjap@deb.uminho.pt (L. Abrunhosa).

1 These authors contributed equally to the supervision of this work.
}

deoxynivalenol and their de-epoxy-forms, zearalenone (ZEA) and its derivative $\alpha$-zearalenol, have been reported (Fink-Gremmels, 2008). Those mycotoxins are thermostable and are not destroyed by dairy processing methods; they remain in pasteurized milk and in fermented dairy products (Iha et al., 2013).

Ingestion of mycotoxins may cause acute mycotoxicosis and several chronic adverse effects, such as mutagenic, carcinogenic, teratogenic, estrogenic and immunosuppressive effects (Paterson and Lima, 2010). The International Agency for Research on Cancer (IARC) classified aflatoxin B1 (AFB1) in group 1 (carcinogenic to humans), OTA and FB1 in group 2B (possibly carcinogenic to humans) and ZEA in group 3 (not classifiable as to its carcinogenicity to humans) (IARC, 2016).

Milk is the primary source of nutrition for growing infants. However, commercial milk and milk products as well as milk of nursing women can be contaminated with mycotoxins. Therefore, infants and young children are more sensitive to these serious problems than adults (Mohammadi, 2011). Studies have reported an association between AFB1 exposure and stunted foetal, infant and child growth in West African countries (Gong et al., 2004). Moreover, diagnosis of nephropathic patients from Tunisia showed that the presence of OTA was linked to a chronic interstitial nephropathy (Zaied et al., 2011). In consideration of the health risks and to ensure food safety, the Joint FAO/WHO Expert 
Committee on Food Additives (JECFA) sets limits for mycotoxin daily intake as follows: ZEA - $0.5 \mu \mathrm{g} / \mathrm{kg}$ body weight per day and OTA $112 \mathrm{ng} / \mathrm{kg}$ body weight per week, and no limits have been indicated regarding tolerable intake for AFB1 because of its carcinogenic effects (Bol et al., 2016).

As a solution to this serious problem, numerous physical, chemical and biological strategies have been reported for mycotoxin detoxification. Recently, several microorganisms were investigated for mycotoxin degradation or adsorption, such as lactic acid bacteria (Abrunhosa et al., 2014; Elsanhoty et al., 2013), yeasts (Petruzzi et al., 2015; Zhang et al., 2016), and other bacteria (Harkai et al., 2016).

Kefir is a fermented dairy product originating from the Caucasus Mountains. The word Kefir "keyif" comes from the Turkish language and means "good feeling" owing to the refreshing nature of this beverage (Leite et al., 2013). It is obtained from lactic-alcoholic fermentation of milk by gelatinous irregular grains, which range from 0.3 to $3.5 \mathrm{~cm}$ in diameter. The colour of the grains varies from white to light yellow, and they resemble tiny florets of cauliflower (Hamet et al., 2013). Kefir grains are a complex symbiotic association of lactic acid bacteria (Lactobacillus kefiri, Lactobacillus kefiranofaciens, Lactobacillus kefirgranum, Lactobacillus parakefiri, Lactobacillus delbrueckii, Lactobacillus acidophilus, Lactobacillus brevis, Lactobacillus helveticus, Lactobacillus casei, Lactobacillus paracasei, Lactobacillus fermentum, Lactobacillus plantarum, and Lactobacillus gasseri), yeasts (Kluyveromyces marxianus, Kluyveromyces lactis, Saccharomyces cerevisiae, Torulaspora delbrueckii, Candida kefir, Pichia fermentans, Kazachstania unispora, and Kazachstania exigua) and acetic acid bacteria that cohabitate in a protein and polysaccharide (kefiran) matrix (Garofalo et al., 2015). Microbial fermentation produces several bioactive compounds, such as peptides, amino acids, bacteriocins, ethanol, $\mathrm{CO}_{2}$, acetaldehyde, acetoin, diacetyl, exopolysaccharides, folic acid, calcium and vitamins B1 and B12, as well as lactic and acetic acid (Garofalo et al., 2015). Due to its high nutritional value and content of natural probiotics, Kefir possesses numerous health benefits (Satir and Guzel-Seydim, 2015), including modulating the immune system and enhancing digestive health, as well as antimicrobial, anti-tumoral and antioxidant activities (Ahmed et al., 2013; Vinderola et al., 2005). A few studies regarding mycotoxin decontamination by Kefir have reported the ability of Kefir grains to reduce $96.8 \%$ of AFG1 at a concentration of $20 \mu \mathrm{g} / \mathrm{kg}$ in pistachio nuts after a $6 \mathrm{~h}$ contact time at $30{ }^{\circ} \mathrm{C}$ (Ansari et al., 2015). In addition, the ability of Kefir grains to bind $91.9 \%$ of AFM1 $(0.5 \mu \mathrm{g} / \mathrm{L})$ in milk has been reported (Isakhani et al., 2014). However, as far as we know, the effect of Kefir microbiota on other mycotoxins has not previously been investigated, nor has any active strain been isolated from it.

The objective of the current study was to investigate the microbiota characteristics of Kefir from Tunisia and to isolate bacteria and/or yeasts with biodegradation and/or adsorption properties for AFB1, OTA and ZEA. Additionally, the main strains involved in the adsorption of mycotoxins studied were identified by DNA sequencing, and the stability of the microorganism/mycotoxin complexes was tested by simulating a $\mathrm{pH}$ change in the gastrointestinal tract.

\section{Materials and methods}

\subsection{Kefir production}

Kefir used in this study was a traditional culture and original grain from Monastir, Tunisia. The preparation of Kefir was as follow: grains $(10 \% \mathrm{w} / \mathrm{v})$ were activated in commercial Ultra-high temperature cows' milk (protein, 3.4\%; fat, 1.6\%; carbohydrate, $5.1 \%$ ) and incubated at $25{ }^{\circ} \mathrm{C}$ for $24 \mathrm{~h}$. The grains were then retrieved by sieving from the cultured milk and rinsed with mild sterile distilled water to remove the clotted milk. Afterwards, the grains were re-inoculated into fresh sterile milk and incubated under the same conditions. The milk was changed every day during the one week incubation period. This step was repeated twice to obtain grains well acclimated to milk and ready for use as starter inocula in the following experiments.

\subsection{Microbiological analysis of Kefir}

Enumeration and isolation of microorganisms from both fermented milk and Kefir grains were performed. After an incubation period of activated grains at $25^{\circ} \mathrm{C}$ for $24 \mathrm{~h}$ in milk, ten millilitres of Kefir fermented milk was homogenized with $90 \mathrm{~mL}$ of a sterile sodium thiosulfate solution $(0.2 \% \mathrm{w} / \mathrm{v})$ for $1 \mathrm{~min}$ using a Stomacher. Then, ten grams of Kefir grains were suspended in $90 \mathrm{~mL}$ of sterile saline (0.85\%) and homogenized with a Stomacher for $20 \mathrm{~min}$. Serial decimal dilutions were prepared in the same diluent, and $0.1 \mathrm{~mL}$ was inoculated in triplicate by surface spreading on specific solid media. Lactic acid bacteria (LAB) were isolated on de Man Rogosa Sharpe agar (MRS) and incubated at $30{ }^{\circ} \mathrm{C}$ under anaerobic conditions for 5 days. Yeasts were isolated on yeast extract peptone dextrose (YPD) agar at $25{ }^{\circ} \mathrm{C}$ for 3 days. MRS agar (Oxoid, Hampshire, UK), and YPD was prepared with $20 \mathrm{~g} / \mathrm{L}$ of bacteriological peptone (Himedia, Mumbai, India), $10 \mathrm{~g} / \mathrm{L}$ of yeast extract (Himedia, Mumbai, India), $20 \mathrm{~g} / \mathrm{L}$ of glucose (Fisher Chemical, Porto Salvo, Portugal) and $20 \mathrm{~g} / \mathrm{L}$ of bacteriological agar (Oxoid, Hampshire, UK). LAB and yeasts were counted, and some strains were isolated, streak-plate purified and examined microscopically. The results of the viable counts were expressed as the means of colony forming units (CFU) per gram or per $\mathrm{mL}$ of Kefir \pm standard deviation. Isolated colonies were grown in MRS broth supplemented with 20\% glycerol and preserved at $-80^{\circ} \mathrm{C}$ until further analyses.

\subsection{Evaluation of mycotoxin-detoxifying properties of Kefir}

The ability of Kefir cultures to biotransform or adsorb mycotoxins in milk was first evaluated. Stock standard solutions of AFB1 (Sigma), OTA (Panreac) and ZEA (Sigma) were prepared in methanol at $1 \mathrm{mg} / \mathrm{mL}$ and stored at $-20{ }^{\circ} \mathrm{C}$ until use. Commercial UHT cows' milk was artificially contaminated with $1 \mu \mathrm{g} / \mathrm{mL}$ of each mycotoxin (AFB1, OTA and ZEA) by adding the appropriate amount of stock standards. Falcon tubes containing $5 \mathrm{~mL}$ of mycotoxin-contaminated milk were prepared in triplicate to study the biotransformation and adsorption properties of Kefir. Kefir grains $(10 \%, w / v)$ were added to tubes containing five millilitres of mycotoxin-contaminated milk. The tubes were mixed and incubated aerobically at $25^{\circ} \mathrm{C}$ for $24 \mathrm{~h}$. Three non-inoculated tubes containing milk contaminated with mycotoxins were also prepared and incubated to be used as negative controls.

After the incubation period, for the determination of mycotoxins, tubes intended to test biotransformation were treated differently from those intended to test adsorption. To test biotransformation, $5 \mathrm{~mL}$ of acetonitrile/methanol/acetic acid (78/20/2, v/v/v) was directly added to tubes, and after a strong vortex mixing for $1 \mathrm{~min}$, they were left to stand overnight at room temperature. Negative controls were also treated in this way. To test adsorption, tubes were first centrifuged at 9000 $\mathrm{RCF}$ for $20 \mathrm{~min}$, the clear liquid fraction transferred to clean tubes and an equal volume of the earlier organic solution was added. After being strongly vortexed for $1 \mathrm{~min}$, they were also left to stand overnight. Thereafter, all samples were filtered into clean $2-\mathrm{mL}$ vials using a syringe filter $(0.2 \mu \mathrm{m}$, Nylon $)$ and preserved at $-20^{\circ} \mathrm{C}$ until HPLC analysis.

\subsection{Evaluation of the mycotoxin-detoxifying properties of strains isolated from Kefir}

After streak-plate purification and microscopic inspection, some of the microorganisms isolated from Kefir were investigated for mycotoxin biotransformation and adsorption properties using culture media and milk. Bacteria and yeasts were propagated respectively in $10 \mathrm{~mL}$ of MRS or YPD broth at $30^{\circ} \mathrm{C}$ for 3 days. Optical density (O.D.) was determined at $600 \mathrm{~nm}$ and adjusted to 2.0 with sterile distilled water. MRS broth, YPD broth and milk supplemented with $1 \mu \mathrm{g} / \mathrm{mL}$ of each mycotoxin 
(AFB1, OTA and ZEA) was prepared as described before. LAB were inoculated $(1 \%, \mathrm{w} / \mathrm{v})$ in MRS broth, yeasts in YPD broth and both were tested in milk containing mycotoxins as described in previous section. Experiments were performed in triplicate. After incubation at $25^{\circ} \mathrm{C}$ for $24 \mathrm{~h}$, mycotoxins were extracted as described previously for biotransformation and adsorption experiments. Samples were filtered and kept at $-20^{\circ} \mathrm{C}$ until HPLC analysis.

\subsection{Identification of relevant strains}

Strains isolated from Kefir that showed better effects for the adsorption of AFB1, OTA and ZEA were grown in their appropriate broth (MRS for lactic acid bacteria, YPD for yeast) and were identified using molecular methods by STAB VIDA laboratory, Portugal. The amplification of 16S rDNA from the bacterial strains was performed with the primers 27F ( $5^{\prime}$-AGAATTTGATCMTGGCTCAG-3') and 1492R (5'-TACGGYTACCTTGTTACGACTT-3'). For yeasts, the fungal ribosomal ITS1-5.8S-ITS2 rDNA region was amplified with ITS1 (5'-TCCGTAGGTGAACCTGCGG-3') and ITS4 (5'TCCTCCGCTTATTGATATGC- $\left.3^{\prime}\right)$; the D1/D2 domain of the $26 \mathrm{~S}$ rDNA region was amplified using the specific primers NL1 (5'-GCATATCAATAAGCGGAGGAAAAG-3') and NL4 (5'GGTCCGTGTTTCAAGACGG-3'). The PCR products of 165 rDNA, ITS15.8S-ITS2 and D1/D2 26S rDNA were sequenced, the obtained sequences were aligned and the percentage of homology with sequences in the GenBank database was determined. The nucleotide sequences of both bacteria and yeasts were submitted to the NCBI database.

\subsection{Stability of the microorganism/mycotoxin complexes at different $\mathrm{pHs}$}

To determine the stability of the microorganism/mycotoxin complexes, pellets of the Kefir consortium and of selected strains with bound mycotoxins were sequentially washed with buffer solutions at $\mathrm{pH} \mathrm{7,} 3$ and 8 to simulate the $\mathrm{pH}$ conditions of the gastrointestinal tract. Experiments were started by adding $5 \mathrm{~mL}$ of $0.1 \mathrm{M}$ phosphate buffer at $\mathrm{pH} 7$ to pellets and by incubating under rotary agitation at 37 ${ }^{\circ} \mathrm{C}$ for $5 \mathrm{~min}$ to simulate the time spent in the mouth compartment. Thereafter, cells were centrifuged $\left(6500 \mathrm{~g}, 10 \mathrm{~min}, 4^{\circ} \mathrm{C}\right)$ and the supernatant was collected to evaluate the amount of mycotoxins released. Then, the pellets were re-suspended in $5 \mathrm{~mL}$ of $0.1 \mathrm{M}$ citrate/phosphate buffer at $\mathrm{pH} 3$, and the tubes were incubated under the same conditions for $2 \mathrm{~h}$ to simulate the conditions of the stomach. Supernatant recovery was performed as described previously, and pellets were re-suspended in $5 \mathrm{~mL}$ of $0.1 \mathrm{M}$ phosphate buffer $\mathrm{pH} 8$ and incubated in the same way for $2 \mathrm{~h}$ to simulate the small intestine compartment. Tubes were centrifuged a final time, and the three supernatants obtained were extracted separately as previously described in Section 2.3. The amount of released mycotoxins was quantified by HPLC, and the percentage of each toxin bound was calculated.

\subsection{Mycotoxin analysis}

Quantification of mycotoxins was performed by high performance liquid chromatography (HPLC) with fluorescence detection. The HPLC system was equipped with a Varian Prostar 210 pump, a Varian Prostar 410 autosampler, a Jasco FP-920 fluorescence detector and a Jones Chromatography 7971 column heater that was maintained at $30^{\circ} \mathrm{C}$. The instrument and chromatographic data were managed by a Varian 850 MIB data system interface and a Galaxie chromatography data system, respectively. An analytical C18 reversed-phase YMC-Pack ODS-AQ column $(250 \times 4.6 \mathrm{~mm}$ I.D., $5 \mu \mathrm{m})$ connected to a pre-column with the same stationary phase was used.

For AFB1 determination, a mixture of deionized water/acetonitrile/ methanol $(3: 1: 1, \mathrm{v} / \mathrm{v} / \mathrm{v})$ was used as the mobile phase at a flow rate of $1.0 \mathrm{~mL} / \mathrm{min}$. The excitation (ex) and emission (em) wavelengths were set at 365 and $435 \mathrm{~nm}$, respectively (Soares et al., 2010). The determination of OTA was performed according to Abrunhosa et al. (2014) at ex: $333 \mathrm{~nm}$ and em: $460 \mathrm{~nm}$. OTA was eluted isocratically with acetonitrile/water/acetic acid (99:99:2, v/v/v) at a flow rate of $1.0 \mathrm{~mL} / \mathrm{min}$. ZEA determination was performed according to Niderkorn et al. (2007). The wavelengths used for excitation and emission were 280 and $460 \mathrm{~nm}$, respectively. The mobile phase was a mixture of methanol/water/acetic acid $(65 / 35 / 1, \mathrm{v} / \mathrm{v})$ pumped at a flow rate of $1.0 \mathrm{~mL} / \mathrm{min}$. Mobile phases were filtered and degassed with a $0.2-\mu \mathrm{m}$ membrane filter (GHP, Gelman). An aliquot of $50 \mu \mathrm{L}$ was injected for analysis. The retention times of AFB1, OTA, and ZEA were approximately $20.3 \mathrm{~min}, 13.5 \mathrm{~min}$ and $19.7 \mathrm{~min}$, respectively. Mycotoxins were identified by comparing the retention times of the peak samples with the standard curves. Standard concentration range was of 0.005$1.0 \mu \mathrm{g} / \mathrm{mL}$. The limit of detection (LOD) and the limit of quantification (LOQ) were calculated with a signal-to-noise ratio of 3:1 and 10:1, respectively. For AFB1, LOD $=0.004 \mu \mathrm{g} / \mathrm{mL}$ and $\mathrm{LOQ}=0.013 \mu \mathrm{g} / \mathrm{mL}$. For OTA, LOD $=0.005 \mu \mathrm{g} / \mathrm{mL}$ and LOQ $=0.017 \mu \mathrm{g} / \mathrm{mL}$. For ZEA, LOD $=$ $0.012 \mu \mathrm{g} / \mathrm{mL}$ and LOQ $=0.040 \mu \mathrm{g} / \mathrm{mL}$. Mean recoveries were $93 \% \pm$ 7.0 for AFB1, $113 \% \pm 6.7$ for OTA, and $103 \% \pm 6.1$ for ZEA.

\subsection{Statistical analysis}

Results were expressed as mean values \pm standard deviation ( $\mathrm{n}=$ 3 ). The statistical analyses were performed using GraphPad Prism version 7.00 for Windows (La Jolla, California, USA). Two-way ANOVA and Dunnett's post-hoc test were performed to test significant differences between microorganisms' effects and controls $(p<0.05)$.

\section{Results}

\subsection{Microbiological analysis of Kefir}

As presented in Table 1, lactic acid bacteria (LAB) were the dominant microbial population in Kefir. Additionally, the number of LAB was higher in grains $\left(9.5 \times 10^{10} \mathrm{CFU} / \mathrm{g}\right)$ than in milk $\left(8.4 \times 10^{7} \mathrm{CFU} / \mathrm{mL}\right)$. Meanwhile, yeast was present in milk $\left(1.5 \times 10^{9} \mathrm{CFU} / \mathrm{mL}\right)$ at considerably higher amounts than in grains $\left(9.2 \times 10^{6} \mathrm{CFU} / \mathrm{g}\right)$.

\subsection{Evaluation of the mycotoxin-detoxifying properties of Kefir}

The biotransformation and adsorption of AFB1, OTA and ZEA by the Kefir microbial consortium in milk are depicted in Table 2. Some elimination of mycotoxins was observed in the biotransformation experiments. The highest percentage of elimination was obtained with AFB1 (31\%), followed by OTA (12\%) and ZEA (10\%). No biotransformation products were detected in the samples. Therefore, it is impossible to definitively state that a chemical transformation of mycotoxins occurred. Rather, it is more likely that the extraction process was not able to completely extract mycotoxins from the biomass formed during the fermentation process. Interestingly, in the adsorption experiments, the Kefir consortium showed better results since $100 \%$ of ZEA, $94 \%$ of OTA and $82 \%$ of AFB1 were eliminated from the liquid fraction. Thus, the Kefir consortium was more efficient at binding mycotoxins than at transforming them.

\section{Table 1}

Lactic acid bacteria and yeast counts in Kefir culture after $24 \mathrm{~h}$ of fermentation in milk. The results are expressed as the means \pm standard deviation of three independent experiments.

\begin{tabular}{lll}
\hline \multirow{2}{*}{ Samples } & \multicolumn{2}{l}{ Viable counts on selective growth media } \\
\cline { 2 - 3 } & MRS & YPD \\
\hline Kefir grain & $(9.5 \pm 0.15) \times 10^{10} \mathrm{CFU} / \mathrm{g}$ & $(9.2 \pm 0.14) \times 10^{6} \mathrm{CFU} / \mathrm{g}$ \\
Kefir milk & $(8.4 \pm 1.03) \times 10^{7} \mathrm{CFU} / \mathrm{mL}$ & $(1.5 \pm 0.45) \times 10^{9} \mathrm{CFU} / \mathrm{mL}$ \\
\hline
\end{tabular}


Table 2

Percentage of AFB1, OTA and ZEA eliminated by the Kefir culture during biotransformation and adsorption experiments conducted in milk (final $\mathrm{pH} 4.8$ ). The results are expressed as the means \pm standard deviation $(n=3)$. All values are significantly different from control experiments $(p<0.05)$.

\begin{tabular}{llll}
\hline & AFB1 & OTA & ZEA \\
\hline Biotransformation (\%) & $31 \pm 3.2$ & $12 \pm 3.3$ & $10 \pm 0.7$ \\
Adsorption (\%) & $82 \pm 3.1$ & $94 \pm 0.8$ & 100 \\
\hline
\end{tabular}

\subsection{Evaluation of the mycotoxin-detoxifying properties of strains isolated from Kefir}

In total, twenty-two strains were isolated from Kefir (grains and milk), among them, 11 Lactobacilli (LAB) and 11 yeasts. Data showed that the biotransformation and adsorption of AFB1, OTA and ZEA were strain and culture medium dependent (Tables 3 and 4). Nonetheless, the binding effect was the most relevant and strong for almost all of the isolates.

According to Table 3, the LAB binding assays for AFB1 in MRS showed no effect ( $0 \%$ ). However, LAB showed the ability to bind OTA (up to 29\%) and ZEA (up to 60\%). Compared to the other isolates, strain KFGM5 showed the highest binding capacity for OTA (29\%), followed by KFLM4C (16\%), KFLM3 and KFGM1 (15\%). Moreover, the ZEA binding capabilities of strains KFLM3, KFGM1 and KFGM5 were the strongest under those conditions because, respectively, $60 \%, 52 \%$ and $46 \%$ adsorption was observed.

The adsorption capability of eleven yeast strains in YPD medium is also presented in Table 3. Yeasts bound small amounts of AFB1 (up to $8 \%$ ) and OTA (up to $6 \%$ ). Strain KFGY2 was able to remove $8 \%$ of AFB1, whereas strains KFGY7 and KFLY6 were able to bind 6\% of OTA. Greater amounts of ZEA were eliminated from medium by yeasts (up to $44 \%$ ). Strain KFLY4 was able to bind 44\% of ZEA and strain KFGY2 bound 31\% and strain KFGY1 bound $29 \%$.

The same experiments were repeated in milk. All of the strains changed their behaviour, and their binding ability improved as presented in Table 4 . The amount of mycotoxins removed in milk reached $80 \%$ for AFB1, 81\% for OTA and 100\% for ZEA, depending on the strain. The strains KFLM3 and KFGY7 showed the highest AFB1 binding ability, with removal percentages of $80 \%$ and $74 \%$, respectively. The highest amounts of OTA bound were obtained with strains KFLM3 (81\%), KFLY6 (74\%) and KFGY7 (62\%). We also observed that six strains, KFLM3, KFLY1, KFLY3, KFLY5, KFGY1 and KFLY6, were able to bind the totality of ZEA present in milk (100\%) and that strain KFGY7 bound $95 \%$ of mycotoxin. These observations suggest that the binding capacity is dependent on the particular characteristics of each strain and mycotoxin type but also that the culture medium used to grow the strains is the most determinant factor.

\subsection{Identification of relevant strains}

Considering the results obtained in previous experiments, three strains (KFLM3, KFGM1 and KFGY7) were selected for further characterization. Two strains of bacteria (KFLM3 and KFGM1) and one strain of yeast (KFGY7) that were able to bind mycotoxins in milk were identified using molecular methods. According to $16 \mathrm{~S}$ rRNA gene sequencing, the isolates KFLM3 and KFGM1 had 100\% nucleotide homology with Lactobacillus kefiri and Acetobacter syzygii, respectively. The yeast strain KFGY7 was identified as Kazachstania servazzii (96\% homology to the GenBank sequences). The $16 \mathrm{~S}$ rRNA sequences of the three strains were deposited in GenBank under the accession numbers: KX987247 for Lactobacillus kefiri KFLM3, KX987248 for Acetobacter syzygii KFGM1 and KX987246 for Kazachstania servazzii KFGY7.

\subsection{Stability of the microorganism/mycotoxin complexes at different $\mathrm{pHs}$}

The identified strains were assessed for microorganism/mycotoxin complex stability by simulating the $\mathrm{pH}$ of the gastrointestinal tract. The experiments involved successive treatment of pellets that had previously been bound to mycotoxins in milk, with buffers at $\mathrm{pH} 7,3$ and 8 . The percentage of mycotoxins released was determined after each treatment and is presented in Table 5. For the Kefir microbial consortium, after treatment at $\mathrm{pH}$ 7, a considerable amount of AFB1 (20\%) and OTA (37\%) were released from pellets, while for ZEA, only $14 \%$

Table 3

Percentage of AFB1, OTA and ZEA eliminated by the strains isolated from Kefir. Biotransformation and adsorption experiments were conducted in MRS and YPD.

\begin{tabular}{|c|c|c|c|c|c|c|c|}
\hline \multirow[t]{2}{*}{ Isolates } & \multirow[t]{2}{*}{ Origin } & \multicolumn{2}{|l|}{ AFB1 } & \multicolumn{2}{|l|}{ OTA } & \multicolumn{2}{|l|}{ ZEA } \\
\hline & & Biotransformation & Adsorption & Biotransformation & Adsorption & Biotransformation & Adsorption \\
\hline \multicolumn{8}{|c|}{ LAB in MRS medium } \\
\hline KFLM2 & Milk & $0^{*}$ & $0^{*}$ & $1 \pm 0.3^{*}$ & $8 \pm 0.7^{*}$ & $9 \pm 1.3$ & $19 \pm 2.4$ \\
\hline KFLM3 & Milk & $\mathbf{0}^{*}$ & $\mathbf{0}^{*}$ & $8 \pm 0.5^{*}$ & $15 \pm 2.6$ & $56 \pm 2.3$ & $60 \pm 4.0$ \\
\hline KFLM4F & Milk & $8 \pm 1.1^{*}$ & $0^{*}$ & $5 \pm 1.6^{*}$ & $10 \pm 1.4$ & $6 \pm 0.8^{*}$ & $12 \pm 1.5$ \\
\hline KFLM4C & Milk & $0^{*}$ & $0^{*}$ & $6 \pm 2.0^{*}$ & $16 \pm 0.4$ & $33 \pm 2.4$ & $40 \pm 2.9$ \\
\hline KFGM1 & Grain & 0* & 0* & $2 \pm 0.2^{*}$ & $15 \pm 0.9$ & $48 \pm 1.0$ & $52 \pm 0.9$ \\
\hline KFGM2 & Grain & $0^{*}$ & $0^{*}$ & $6 \pm 3.2^{*}$ & $10 \pm 1.7$ & $12 \pm 0.7$ & $14 \pm 1.7$ \\
\hline KFGM3 & Grain & $0^{*}$ & $0^{*}$ & $3 \pm 0.5^{*}$ & $9 \pm 2.1^{*}$ & $8 \pm 2.7^{*}$ & $16 \pm 1.4$ \\
\hline KFGM4 & Grain & $17 \pm 2.3$ & $0.2 \pm 0.1^{*}$ & $6 \pm 0.8^{*}$ & $8 \pm 0.4^{*}$ & $9 \pm 3.1$ & $13 \pm 1.8$ \\
\hline KFGM5 & Grain & $0^{*}$ & $0^{*}$ & $44 \pm 3.2$ & $29 \pm 0.9$ & $71 \pm 2.8$ & $46 \pm 2.6$ \\
\hline KFGM7 & Grain & $0^{*}$ & $0^{*}$ & $0^{*}$ & $1 \pm 0.4^{*}$ & $10 \pm 1.4$ & $9 \pm 0.5^{*}$ \\
\hline KFGM8 & Grain & $0^{*}$ & $0^{*}$ & $0^{*}$ & $7 \pm 2.7^{*}$ & $17 \pm 1.8$ & $25 \pm 2.3$ \\
\hline \multicolumn{8}{|c|}{ Yeasts in YPD medium } \\
\hline KFLY1 & Milk & $0^{*}$ & $4 \pm 1.0^{*}$ & $0^{*}$ & $0^{*}$ & $0^{*}$ & $1 \pm 0.3^{*}$ \\
\hline KFLY3 & Milk & $0^{*}$ & $3 \pm 0.8^{*}$ & $0^{*}$ & $0^{*}$ & $0^{*}$ & $8 \pm 2.4^{*}$ \\
\hline KFLY4 & Milk & $1 \pm 0.9^{*}$ & $2 \pm 0.6^{*}$ & $0^{*}$ & $0^{*}$ & $0^{*}$ & $44 \pm 5.0$ \\
\hline KFLY5 & Milk & $0^{*}$ & $5 \pm 2.1^{*}$ & $0^{*}$ & $0^{*}$ & $10 \pm 0.8$ & $12 \pm 1.9$ \\
\hline KFLY6 & Milk & $0^{*}$ & $0^{*}$ & $4 \pm 0.4^{*}$ & $6 \pm 3.8^{*}$ & $8 \pm 0.7^{*}$ & $10 \pm 1.2$ \\
\hline KFGY1 & Grain & $20 \pm 3.2$ & $7 \pm 1.3^{*}$ & $16 \pm 2.7$ & $5 \pm 1.0^{*}$ & $32 \pm 2.4$ & $29 \pm 0.7$ \\
\hline KFGY2 & Grain & $5 \pm 2.5$ & $8 \pm 0.9^{*}$ & $0^{*}$ & $0^{*}$ & $10 \pm 1.6$ & $31 \pm 1.5$ \\
\hline KFGY4 & Grain & $0^{*}$ & $0^{*}$ & $0^{*}$ & $0^{*}$ & $0^{*}$ & $0^{*}$ \\
\hline KFGY5 & Grain & $0^{*}$ & $0.5 \pm 0.2^{*}$ & $0^{*}$ & $0^{*}$ & $0^{*}$ & $0^{*}$ \\
\hline KFGY6 & Grain & $0.5 \pm 0.4^{*}$ & $3 \pm 1.1^{*}$ & $0^{*}$ & $0^{*}$ & $0^{*}$ & $23 \pm 2.1$ \\
\hline KFGY7 & Grain & $\mathbf{0}^{*}$ & $\mathbf{0}^{*}$ & $\mathbf{0}^{*}$ & $6 \pm 2.6^{*}$ & $9 \pm 1.4$ & $18 \pm 0.9$ \\
\hline
\end{tabular}

The results are expressed as the means \pm standard deviation $(n=3)$. Strains highlighted in bold were selected for the following experiments.

* Values are not significantly different from control experiments $(p<0.05)$. 
Table 4

Percentage of AFB1, OTA and ZEA eliminated by the Kefir strains in biotransformation and adsorption experiments conducted in milk.

\begin{tabular}{|c|c|c|c|c|c|c|c|}
\hline \multirow[t]{2}{*}{ Isolates } & \multirow[t]{2}{*}{ Origin } & \multicolumn{2}{|l|}{ AFB1 } & \multicolumn{2}{|l|}{ OTA } & \multicolumn{2}{|l|}{ ZEA } \\
\hline & & Biotransformation & Adsorption & Biotransformation & Adsorption & Biotransformation & Adsorption \\
\hline KFLM2 & Milk & $5 \pm 0.6^{*}$ & $29 \pm 3.4$ & $0^{*}$ & $7 \pm 1.4^{*}$ & $1 \pm 0.4^{*}$ & $23 \pm 2.4$ \\
\hline KFLM3 & Milk & $4 \pm 0.3^{*}$ & $80 \pm 2.1$ & $\mathbf{0}^{*}$ & $81 \pm 2.7$ & $7 \pm 1.3^{*}$ & 100 \\
\hline KFLM4F & Milk & $7 \pm 0.8^{*}$ & $24 \pm 1.5$ & $0^{*}$ & $8 \pm 1.3^{*}$ & $0^{*}$ & $24 \pm 1.4$ \\
\hline KFLM4C & Milk & $1 \pm 0.6^{*}$ & $22 \pm 0.7$ & $0^{*}$ & $10 \pm 2.8$ & $0^{*}$ & $20 \pm 1.6$ \\
\hline KFGM1 & Grain & $2 \pm 0.4^{*}$ & $65 \pm 1.6$ & $\mathbf{0}^{*}$ & $50 \pm 0.7$ & $\mathbf{0}^{*}$ & $64 \pm 2.3$ \\
\hline KFGM2 & Grain & $1 \pm 0.5^{*}$ & $28 \pm 2.3$ & $0^{*}$ & $12 \pm 0.3$ & $0^{*}$ & $19 \pm 2.2$ \\
\hline KFGM3 & Grain & $0^{*}$ & $35 \pm 1.9$ & $0^{*}$ & $11 \pm 1.5$ & $0^{*}$ & $13 \pm 2.0$ \\
\hline KFGM4 & Grain & $1 \pm 0.3^{*}$ & $7 \pm 1.1^{*}$ & $4 \pm 0.9^{*}$ & $8 \pm 2.3^{*}$ & $0^{*}$ & $0^{*}$ \\
\hline KFGM5 & Grain & $0^{*}$ & $0^{*}$ & $0^{*}$ & $0^{*}$ & $0^{*}$ & $0^{*}$ \\
\hline KFGM7 & Grain & $0^{*}$ & $31 \pm 2.7$ & $0^{*}$ & $16 \pm 2.1$ & $0^{*}$ & $25 \pm 1.5$ \\
\hline KFGM8 & Grain & $2 \pm 0.9^{*}$ & $20 \pm 2.5$ & $0^{*}$ & $6 \pm 1.9^{*}$ & $0^{*}$ & $17 \pm 1.3$ \\
\hline KFLY1 & Milk & $0^{*}$ & $61 \pm 3.2$ & $0^{*}$ & $53 \pm 3.6$ & $0^{*}$ & 100 \\
\hline KFLY3 & Milk & $7 \pm 0.8^{*}$ & $72 \pm 1.9$ & $0^{*}$ & $54 \pm 2.7$ & $0^{*}$ & 100 \\
\hline KFLY4 & Milk & $6 \pm 0.7^{*}$ & $29 \pm 1.8$ & $0^{*}$ & $8 \pm 1.6^{*}$ & $0^{*}$ & $26 \pm 2.0$ \\
\hline KFLY5 & Milk & $1 \pm 0.5^{*}$ & $64 \pm 2.2$ & $0^{*}$ & $55 \pm 2.4$ & $0^{*}$ & 100 \\
\hline KFLY6 & Milk & $3 \pm 0.9^{*}$ & $70 \pm 5.6$ & $0^{*}$ & $74 \pm 3.5$ & $3 \pm 0.4^{*}$ & 100 \\
\hline KFGY1 & Grain & $15 \pm 1.2$ & $71 \pm 3.5$ & $0^{*}$ & $42 \pm 2.6$ & $0^{*}$ & 100 \\
\hline KFGY2 & Grain & $8 \pm 1.0^{*}$ & $20 \pm 3.4$ & $0^{*}$ & $0^{*}$ & $0^{*}$ & $1 \pm 0.5^{*}$ \\
\hline KFGY4 & Grain & $0^{*}$ & $65 \pm 2.8$ & $0^{*}$ & $44 \pm 1.8$ & $0^{*}$ & $82 \pm 3.4$ \\
\hline KFGY5 & Grain & $8 \pm 1.5^{*}$ & $7 \pm 1.0^{*}$ & $0^{*}$ & $0^{*}$ & $0^{*}$ & $0^{*}$ \\
\hline KFGY6 & Grain & $1 \pm 0.4^{*}$ & $32 \pm 4.3$ & $0^{*}$ & $1 \pm 0.6^{*}$ & $0^{*}$ & $5 \pm 0.8^{*}$ \\
\hline KFGY7 & Grain & $31 \pm 2.3$ & $74 \pm 2.1$ & $11 \pm 2.0$ & $62 \pm 2.4$ & $22 \pm 2.2$ & $95 \pm 5.2$ \\
\hline
\end{tabular}

The results are expressed as the means \pm standard deviation $(n=3)$. Strains highlighted in bold were selected for the following experiments

$*$ Values are not significantly different from control experiments $(p<0.05)$.

was detected in solution. After treatment at $\mathrm{pH} 3$, small amounts of bound AFB1 (6\%), OTA (5\%) and ZEA (0\%) were released from the Kefir consortium. Nonetheless, with the treatment at $\mathrm{pH} 8$, further amounts of AFB1 (14\%), OTA (14\%) and ZEA (51\%) were released. In total, 40,56 and $65 \%$ of AFB1, OTA and ZEA were, respectively, released from the Kefir culture biomass.

Concerning the strains isolated from Kefir, the results show that the microorganism/mycotoxin complexes are more sensitive at $\mathrm{pH} 3$ and more stable at $\mathrm{pH} 7$ and 8. Strain L. kefiri KFLM3 released, after the three treatments, a considerable amount of AFB1 (66\%), OTA (74\%) and ZEA (46\%). Similarly, strain A. syzygii KFGM1 released 64\% of AFB1, $65 \%$ of OTA and 55\% of ZEA. Compared to bacteria, the yeast $K$. servazzii KFGY7 showed the most stable microorganism/mycotoxin complexes. As presented in Table 5, K. servazzii KFGY7 only released $35 \%$ of AFB1, 31\% of OTA and 33\% of ZEA. In summary, KFGY7 was the most effective among the three tested strains.

\section{Discussion}

In Kefir grains, a high number of microorganisms involved in milk fermentation cohabitate. Thus, the enumeration of Kefir strains was essential to ensure their viability after the fermentation process. In the present work, microbial analyses of both Kefir grains and Kefir fermented milk were performed. The counts of viable LAB in Kefir grains were similar to those reported for Italian Kefir grains, which ranged between $2.4 \times 10^{7}$ and $1.1 \times 10^{9} \mathrm{CFU} / \mathrm{g}$ (Garofalo et al., 2015). The same was observed with counts of viable yeasts (Garofalo et al., 2015; Gulitz et al., 2011). However, the species that constitute the Kefir consortium may differ in regard to the origin of the Kefir grains and nature of the milk used (Garofalo et al., 2015). It has been reported that the Lactobacillus spp., Lactococcus spp. and Bifidobacterium spp. contents were higher in Kefir made with goat milk than that made with cow milk (Satir and Guzel-Seydim, 2015).

The evaluation of the mycotoxin-detoxification properties of the Kefir consortium in milk showed that it was able to bind $82 \%, 94 \%$ and $100 \%$ of AFB1, OTA and ZEA, respectively. To the best of our knowledge, there is no previous report concerning the ability of the Kefir consortium to bind these mycotoxins. Only some references are found concerning AFM1 (Sani et al., 2014). Because Kefir is a rich ecosystem containing complex microflora, it was crucial to investigate the main isolates implicated in the mycotoxin adsorption process. Thus, Kefir $\mathrm{LAB}$ and yeast isolates were evaluated for their mycotoxin binding potential in MRS and YPD medium, respectively. As depicted in Table 3, LAB showed no effect on AFB1 binding (0\%) in MRS. These data are in disagreement with data obtained by other authors, who reported the ability of Lactobacillus rhamnosus strains to bind 77-95\% AFB1 (Turbic et al., 2002). OTA binding by LAB isolates from Kefir was also assessed, and the results showed toxin binding of $1-29 \%$. A previous report showed that $L$. plantarum could bind $12-56 \%$ of OTA (Piotrowska and Zakowska, 2005). Similarly, Turbic et al. (2002) reported that 36-76\% of OTA was bound by L. rhamnosus strains. Moreover, all of the LAB isolates from our Kefir demonstrated their ability to remove ZEA from MRS medium, with a percentage ranging between $9 \%$ and $60 \%$. Those observations are in agreement with those of Zhao et al. (2015), who reported

Table 5

Percentage of AFB1, OTA and ZEA recovered from microorganism cells after incubation with buffer solutions at pH 7, 3 and 8 .

\begin{tabular}{|c|c|c|c|c|c|c|c|c|c|c|c|c|}
\hline \multirow[t]{2}{*}{ Isolates } & \multicolumn{4}{|l|}{ AFB1 } & \multicolumn{4}{|l|}{ OTA } & \multicolumn{4}{|l|}{ ZEA } \\
\hline & pH 7 & pH 3 & pH 8 & Total & pH 7 & pH 3 & pH 8 & Total & pH 7 & pH 3 & pH 8 & Total \\
\hline Kefir & $20 \pm 1.5$ & $6 \pm 0.3$ & $14 \pm 1.5$ & $40 \pm 2.6$ & $37 \pm 2.5$ & $5 \pm 0.7$ & $14 \pm 1.8$ & $56 \pm 4.7$ & $14 \pm 0.7$ & $0^{*}$ & $51 \pm 2.3$ & $65 \pm 2.7$ \\
\hline KFLM3 & $12 \pm 0.5$ & $37 \pm 2.4$ & $17 \pm 1.3$ & $66 \pm 4.1$ & $4 \pm 0.5^{*}$ & $45 \pm 2.6$ & $25 \pm 2.3$ & $74 \pm 5.3$ & $4 \pm 0.6$ & $19 \pm 1.6$ & $23 \pm 1.9$ & $46 \pm 4.0$ \\
\hline KFGM1 & $8 \pm 0.8$ & $51 \pm 2.0$ & $5 \pm 0.5$ & $64 \pm 3.1$ & $4 \pm 0.4^{*}$ & $52 \pm 3.2$ & $9 \pm 0.6$ & $65 \pm 4.2$ & $6 \pm 1.0$ & $38 \pm 1.5$ & $11 \pm 1.2$ & $55 \pm 3.5$ \\
\hline KFGY7 & $5 \pm 0.4$ & $28 \pm 1.8$ & $2 \pm 0.4^{*}$ & $35 \pm 2.6$ & $2 \pm 0.7^{*}$ & $26 \pm 1.2$ & $3 \pm 0.4^{*}$ & $31 \pm 2.5$ & $5 \pm 0.8$ & $22 \pm 1.0$ & $6 \pm 0.7$ & $33 \pm 2.5$ \\
\hline
\end{tabular}

The results are expressed as the means \pm standard deviation $(n=3)$.

* Values are not significantly different from control experiments $(p<0.05)$. 
binding of ZEA in MRS by L. plantarum. Sangsila et al. (2016) also found that $83 \%$ of ZEA was removed by Lactobacillus pentosus from a sodium acetate solution.

In the present work, none of the yeasts isolated from Kefir showed a substantial effect in binding AFB1 (0-8\%) or OTA (0-6\%) in YPD medium. However, moderate ZEA adsorption was noted (0-44\%). Recently, Petruzzi et al. (2015) demonstrated that OTA can be bound by $S$. cerevisiae W46 in substantial amounts (43-76\%). Despite the low binding percentage obtained under these conditions, we noted that the mycotoxin binding ability was extremely dependent on strain and mycotoxin type. A variation in the binding ability between strains was also reported by Peltonen et al. (2001), which considered that dissimilarities in the bacterial cell wall composition were the reason for the different AFB1-binding capabilities of the tested strains. The number of binding sites available probably differs from strain to strain.

At this point, it was observed that the Kefir consortium adsorbed mycotoxins in milk very well, but that the isolated strains added a limited adsorption capacity in culture medium. In addition, experiments were carried on the isolated strains out in milk to verify whether the composition of the medium could have influenced the binding capability of the isolates. Remarkably, some of the Kefir isolates modified their binding behaviour and showed a higher adsorption potential for the tested mycotoxins in milk than in MRS or YPD medium. In milk, some of the isolates were able to remove up to $80 \%$ of AFB1, up to $81 \%$ of OTA and up to $100 \%$ of ZEA. The growth of microorganisms in milk was probably faster than in MRS and YPD, leading to the formation of more biomass and, consecutively, to higher binding percentages. In addition to growth, it is also possible that the nutritional composition of milk could favour the biosynthesis of cell wall components involved in the binding of the mycotoxins. After this characterization, some of the best isolates were identified to species level. Based on DNA sequencing, strains KFGM1 and KFGY7, isolated from Kefir grains, were identified as A. syzygii and K. servazzii, respectively; strain KFLM3, isolated from Kefir fermented milk, was identified as L. kefiri. Their nucleotide sequences were deposited in the National Center for Biotechnology Information (NCBI) under the accession numbers KX987248, KX987246 and KX987247, respectively.

Our results showed that $L$. kefiri KFLM3 was the most effective strain as it bound $80 \%, 81 \%$ and $100 \%$ of AFB1, OTA and ZEA, respectively, in milk. On the other hand, the bacteria A. syzygy KFGM1 bound $65 \%$ AFB1, 50\% OTA and 64\% ZEA, and the yeast $K$. servazzii KFGY7 reduced AFB1, OTA and ZEA in milk by $74 \%, 62 \%$ and $95 \%$, respectively. Corassin et al. (2013) also found that a mixture of lactic acid bacteria (L. rhamnosus, L. delbrueckii subsp. bulgaricus and Bifidobacterium lactis) and S. cerevisiae could completely remove AFM1 from UHT skim milk. Our findings are also in agreement with those of El Khoury et al. (2011), who reported that L. bulgaricus and Streptococcus thermophilus were able to bind AFM1 in skim milk better than in PBS.

The evaluation of the stability of the microorganism/mycotoxin complex is important for predicting the release of mycotoxins during gastrointestinal passage and consequently for estimating the real absorption potential of mycotoxins. Thus, after the binding assays were performed in milk, the obtained pellets (Kefir consortium and selected strains) were assessed for complex stability by treatment with buffers that had different $\mathrm{pHs}$ to simulate the $\mathrm{pH}$ of the gastrointestinal tract. Our results showed that the binding of mycotoxins by the Kefir consortium was moderate, as $40 \%$ of AFB $1,56 \%$ of OTA and $65 \%$ of ZEA were released into buffer after the successive treatments. Nevertheless, the Kefir consortium/mycotoxin complexes were quite stable at $\mathrm{pH} 3$ (no ZEA was released, while only $5 \%$ and $6 \%$ of OTA and AFB1 were, respectively, released).

The bacteria L. kefiri KFLM3 and A. syzygii KFGM1 performed similarly to the Kefir consortium for ZEA, but retained less AFB1 and OTA. They released $66 \%$ and $64 \%$ of AFB 1 and $74 \%$ and $65 \%$ of OTA. In this case, the most favourable $\mathrm{pH}$ for the bacteria/mycotoxin complexes was $\mathrm{pH} 7$, and OTA was the least retained mycotoxin. ZEA binding by the two bacteria was most stable since only $46 \%$ and $55 \%$ of this mycotoxin was released. Zhao et al. (2015) reported that ZEA adsorption onto $L$. plantarum cell walls was also partially reversible and that a small amount of the toxin was detected in the washing solution. Our findings are also in accordance with those of Haskard et al. (2001), who reported reversible binding of aflatoxins to probiotic bacteria. Other authors reported partially reversible AFB1 binding with $L$. casei, L. rhamnosus and L. amylovorus (Hernandez-Mendoza et al., 2009; Peltonen et al., 2001). By contrast, a stable AFM1-LAB (L. rhamnosus and L. plantarum) complex was reported by Elsanhoty et al. (2014).

For the yeast K. servazzii KFGY7, more stable complexes were observed with the mycotoxins studied than for the Kefir consortium, $L$. kefiri KFLM3 and A. syzygii KFGM1. Only 35\% of AFB1, 31\% of OTA and $33 \%$ of ZEA were released after the treatments. This means that, on average, the yeast was able to retain approx. 52\% of the mycotoxins added to milk, which was $2.6 \mu \mathrm{g}$. In this case, $\mathrm{pH} 3$ was less favourable for the retention of mycotoxins, while $\mathrm{pH} 7$ and 8 had almost no effect on the stability of the yeast/mycotoxin complexes since $<6 \%$ of mycotoxin was released with those treatments. Petruzzi et al. (2015) reported that high amounts of OTA (80-85\%) were released by S. cerevisiae after 3 washes of 5 min with an acidic PBS solution (pH 3.5). Later, Petruzzi et al. (2016) tested yeasts in simulated gastrointestinal conditions and found they could retain $21-30 \%$ of OTA after a sequential exposure of $6 \mathrm{~h}$ and $5 \mathrm{~min}$ to salivary, gastric, and intestinal simulated juices. In our case, to simulate the passage time in the gastrointestinal tract, we performed washings only with buffers but the total contact time was of $4 \mathrm{~h}$ and $5 \mathrm{~min}$. Therefore, it is expected that, in vivo, this yeast can retain mycotoxins for a sufficient time to be expelled via faeces. It also should be noted that the real intake levels of the mycotoxins via food should be less than the concentrations studied in this work. In future experiments, this yeast will be tested in conditions that simulate the gastrointestinal tract to confirm its potential.

In summary, the mycotoxin binding process of the Kefir consortium and its isolates was partially reversible, showing a moderate non-covalent interaction between toxins and microorganisms. Moreover, as described by Bevilacqua et al. (2014), the amount of mycotoxin released into the medium was proportional to the number of treatments performed. The percentage of mycotoxins released was also dependent on the strain, buffer $\mathrm{pH}$ and mycotoxin type.

The binding characteristics of a strain are possibly dependent on the exopolysaccharides produced by the microorganisms or on their cell wall composition. LAB from Kefir are able to produce kefiran (Paiva et al., 2016). This exopolysaccharide is a hydrosoluble glucogalactan that is mainly produced by L. kefiranofaciens (Ahmed et al., 2013). Kefiran can form a transparent, flexible, homogeneous and extremely thin edible film that acts as a barrier due to its mechanical properties (Piermaria et al., 2009), and it is possible that this exopolysaccharide may be involved in the adsorption properties of L. kefiri KFLM3. In the case of the yeast $K$. servazzii KFGY7, $\beta$ - $(1,3$ and 1,6)-D-glucans of the cell wall are possibly primarily responsible for the observed adsorption effects since those glucans were also implicated in the mycotoxin-adsorbing properties of S. cerevisiae (Piotrowska and Masek, 2015; Yiannikouris et al., 2006).

\section{Conclusions}

It can be concluded from this study that Kefir grains and their isolates (L. kefiri KFLM3, A. syzygii KFGM1 and K. servazzii KFGY7) are able to bind considerable amounts of mycotoxins AFB1, OTA and ZEA in milk. However, the mycotoxin binding process of the Kefir grains and of the three isolates was reversible, and different quantities of toxin were released after exposure to successive $\mathrm{pH}$ treatments. Even so, on average, the yeast $K$. servazzii KFGY7 was able to retain approx. $52 \%$ of the mycotoxins added to milk, L. kefiri KFLM3 retained approx. $34 \%$ and A. syzygii KFGM1 retained 23\%. The Kefir consortium was able to retain approx. $42 \%$ of the mycotoxins. Thus, our findings support 
that Kefir consumption can naturally help to avoid the deleterious effects of consumed mycotoxins. The isolated strains may be of interest for biotechnological applications in the dairy and feed industries as a novel source of mycotoxin-adsorbing microorganisms.

\section{Acknowledgements}

This study was supported by the Portuguese Foundation for Science and Technology (FCT) under the scope of the strategic funding of the UID/BIO/04469/2013 unit and COMPETE 2020 (POCI-01-0145-FEDER006684), by the BioTecNorte operation (NORTE-01-0145-FEDER000004) funded by the European Regional Development Fund under the scope of Norte2020 - Programa Operacional Regional do Norte and by the Project RECI/BBB-EBI/0179/2012 (FCOMP-01-0124-FEDER027462). Luís Abrunhosa was supported by a grant, UMINHO/BPD/51/ 2015, from the project UID/BIO/04469/2013 financed by FCT/MEC (OE).

\section{References}

Abrunhosa, L., Ines, A., Rodrigues, A.I., Guimaraes, A., Pereira, V.L., Parpot, P., Mendes-Faia, A., Venancio, A., 2014. Biodegradation of ochratoxin A by Pediococcus parvulus isolated from Douro wines. Int. J. Food Microbiol. 188, 45-52.

Ahmed, Z., Wang, Y., Ahmad, A., Khan, S.T., Nisa, M., Ahmad, H., Afreen, A., 2013. Kefir and health: a contemporary perspective. Crit. Rev. Food Sci. Nutr. 53, 422-434.

Ansari, F., Khodaiyan, F., Rezaei, K., Rahmani, A., 2015. Modelling of aflatoxin G1 reduction by kefir grain using response surface methodology. J. Environ. Health Sci. Eng. 13, 1-7.

Bevilacqua, A., Petruzzi, L., Corbo, M.R., Baiano, A., Garofalo, C., Sinigaglia, M., 2014. Ochratoxin A released back into the medium by Saccharomyces cerevisiae as a function of the strain, washing medium and fermentative conditions. J. Sci. Food Agric. 94, 3291-3295.

Bol, E.K., Araujo, L., Veras, F.F., Welke, J.E., 2016. Estimated exposure to zearalenone, ochratoxin $\mathrm{A}$ and aflatoxin $\mathrm{B} 1$ through the consume of bakery products and pasta considering effects of food processing. Food Chem. Toxicol. 89, 85-91.

CAST, 1989. Mycotoxins: Economic and Health Risks. Task Force Report no. 116. Council for Agricultural Science and Technology, Ames, Iowa, p. 91.

Corassin, C.H., Bovo, F., Rosim, R.E., Oliveira, C.A.F., 2013. Efficiency of Saccharomyces cerevisiae and lactic acid bacteria strains to bind aflatoxin M-1 in UHT skim milk Food Control 31, 80-83.

El Khoury, A., Atoui, A., Yaghi, J., 2011. Analysis of aflatoxin M1 in milk and yogurt and AFM1 reduction by lactic acid bacteria used in Lebanese industry. Food Control 22, 1695-1699.

Elsanhoty, R.M., Ramadan, M.F., El-Gohery, S.S., Abol-Ela, M., Azeke, M., 2013. Ability of selected microorganisms for removing aflatoxins in vitro and fate of aflatoxins in contaminated wheat during baladi bread baking. Food Control 33, 287-292.

Elsanhoty, R.M., Salam, S.A., Ramadan, M.F., Badr, F.H., 2014. Detoxification of aflatoxin M1 in yoghurt using probiotics and lactic acid bacteria. Food Control 43, 129-134.

Fink-Gremmels, J., 2008. Mycotoxins in cattle feeds and carry-over to dairy milk: a review. Food Addit. Contam. 25, 172-180.

Garofalo, C., Osimani, A., Milanović, V., Aquilanti, L., De Filippis, F., Stellato, G., Di Mauro, S Turchetti, B., Buzzini, P., Ercolini, D., Clementi, F., 2015. Bacteria and yeast microbiota in milk kefir grains from different Italian regions. Food Microbiol. 49, 123-133.

Gong, Y., Hounsa, A., Egal, S., Turner, P.C., Sutcliffe, A.E., Hall, A.J., Cardwell, K., Wild, C.P., 2004. Postweaning exposure to aflatoxin results in impaired child growth: a longitudinal study in Benin, West Africa. Environ. Health Perspect. 1334-1338.

Gulitz, A., Stadie, J., Wenning, M., Ehrmann, M.A., Vogel, R.F., 2011. The microbial diversity of water kefir. Int. J. Food Microbiol. 151, 284-288.

Hamet, M.F., Londero, A., Medrano, M., Vercammen, E., Van Hoorde, K., Garrote, G.L., Huys, G., Vandamme, P., Abraham, A.G., 2013. Application of culture-dependent and culture-independent methods for the identification of Lactobacillus kefiranofaciens in microbial consortia present in kefir grains. Food Microbiol. 36, 327-334.

Harkai, P., Szabó, I., Cserháti, M., Krifaton, C., Risa, A., Radó, J., Balázs, A., Berta, K., Kriszt, B. 2016. Biodegradation of aflatoxin-B1 and zearalenone by Streptomyces sp. collection. Int. Biodeterior. Biodegrad. 108, 48-56.

Haskard, C.A., El-Nezami, H.S., Kankaanpää, P.E., Salminen, S., Ahokas, J.T., 2001. Surface binding of aflatoxin B1 by lactic acid bacteria. Appl. Environ. Microbiol. 67, 3086-3091.

Hernandez-Mendoza, A., Garcia, H., Steele, J., 2009. Screening of Lactobacillus casei strains for their ability to bind aflatoxin B1. Food Chem. Toxicol. 47, 1064-1068.
IARC (International Agency for Research on Cancer), 2016. Agents Classified by the IARC Monographs. vol. 1-116 Available from:. http://monographs.iarc.fr/ENG/ Classification/latest_classif.php.

Iha, M.H., Barbosa, C.B., Okada, I.A., Trucksess, M.W., 2013. Aflatoxin M1 in milk and distribution and stability of aflatoxin M1 during production and storage of yoghurt and cheese. Food Control 29, 1-6.

Isakhani, S., Marhamatizade, M.H., Ebrahimi, T.M., 2014. The assessment of reducing aflatoxin M1 in kefir by Saccharomyces kefir and Lactobacillus casei TD4 by ELISA method. Trends Life Sci. 3, 268-274.

Leite, A., Leite, D., Del Aguila, E., Alvares, T., Peixoto, R., Miguel, M., Silva, J., Paschoalin, V., 2013. Microbiological and chemical characteristics of Brazilian kefir during fermentation and storage processes. J. Dairy Sci. 96, 4149-4159.

Mohammadi, H., 2011. A review of aflatoxin M1, milk, and milk products. In: GuevaraGonzalez, R.G. (Ed.), Aflatoxins - Biochemistry and Molecular Biology. INTECH, pp. 397-414.

Monbaliu, S., Van Poucke, C., Detavernier, C.l., Dumoulin, F., Van De Velde, M., Schoeters, E., Van Dyck, S., Averkieva, O., Van Peteghem, C., De Saeger, S., 2009. Occurrence of mycotoxins in feed as analyzed by a multi-mycotoxin LC-MS/MS method. J. Agric. Food Chem. 58, 66-71.

Niderkorn, V., Morgavi, D.P., Pujos, E., Tissandier, A., Boudra, H., 2007. Screening of fermentative bacteria for their ability to bind and biotransform deoxynivalenol, zearalenone and fumonisins in an in vitro simulated corn silage model. Food Addit. Contam. 24, 406-415.

Paiva, I.M.d., Steinberg, R.d.S., Lula, I.S., Souza-Fagundes, E.M.D., Mendes, T.d.O., Bell, M.J.V., Nicoli, J.R., Nunes, Á.C., Neumann, E., 2016. Lactobacillus kefiranofaciens and Lactobacillus satsumensis isolated from Brazilian kefir grains produce alpha-glucans that are potentially suitable for food applications. LWT Food Sci. Technol. Int. 72, 390-398.

Paterson, R.R., Lima, N., 2010. Toxicology of mycotoxins. In: Luch, A. (Ed.), Molecular, Clinical and Environmental Toxicology. Birkhauser Verlag, Basel, pp. 31-63.

Peltonen, K., El-Nezami, H., Haskard, C., Ahokas, J., Salminen, S., 2001. Aflatoxin B1 binding by dairy strains of lactic acid bacteria and bifidobacteria. J. Dairy Sci. 84, 2152-2156.

Petruzzi, L., Corbo, M.R., Baiano, A., Beneduce, L., Sinigaglia, M., Bevilacqua, A., 2015. In vivo stability of the complex ochratoxin A - Saccharomyces cerevisiae starter strains. Food Control 50, 516-520.

Petruzzi, L., Corbo, M.R., Sinigaglia, M., Bevilacqua, A., 2016. Ochratoxin A removal by yeasts after exposure to simulated human gastrointestinal conditions. J. Food Sci. 81, M2756-M2760.

Piermaria, J.A., Pinotti, A., Garcia, M.A., Abraham, A.G., 2009. Films based on kefiran, an exopolysaccharide obtained from kefir grain: development and characterization. Food Hydrocoll. 23, 684-690.

Piotrowska, M., Masek, A., 2015. Saccharomyces cerevisiae cell wall components as tools for ochratoxin A decontamination. Toxins 7, 1151-1162.

Piotrowska, M., Zakowska, Z., 2005. The elimination of ochratoxin A by lactic acid bacteria strains. Pol. J. Microbiol. 54, 279-286.

Sangsila, A., Faucet-Marquis, V., Pfohl-Leszkowicz, A., Itsaranuwat, P., 2016. Detoxification of zearalenone by Lactobacillus pentosus strains. Food Control 62, 187-192.

Sani, A.M., Marhamati, Z., Marhamatizade, M., 2014. Bio-detoxification of aflatoxin M1 in kefir using Lactobacillus casei. BioTechnol.: Indian J. 9, 219-224.

Satir, G., Guzel-Seydim, Z.B., 2015. Influence of kefir fermentation on the bioactive substances of different breed goat milks. LWT Food Sci. Technol. Int. 63, 852-858.

Soares, C., Rodrigues, P., Freitas-Silva, O., Abrunhosa, L., Venancio, A., 2010. HPLC method for simultaneous detection of aflatoxins and cyclopiazonic acid. World Mycotoxin J. 3, 225-231.

Turbic, A., Ahokas, J.T., Haskard, C.A., 2002. Selective in vitro binding of dietary mutagens, individually or in combination, by lactic acid bacteria. Food Addit. Contam. 19, 144-152.

Vinderola, C.G., Duarte, J., Thangavel, D., Perdigon, G., Farnworth, E., Matar, C., 2005. Immunomodulating capacity of kefir. J. Dairy Res. 72, 195-202.

Yiannikouris, A., Andre, G., Poughon, L., Francois, J., Dussap, C.G., Jeminet, G., Bertin, G., Jouany, J.P., 2006. Chemical and conformational study of the interactions involved in mycotoxin complexation with beta-D-glucans. Biomacromolecules 7, 1147-1155.

Zaied, C., Bouaziz, C., Azizi, I., Bensassi, F., Chour, A., Bacha, H., Abid, S., 2011. Presence of ochratoxin A in Tunisian blood nephropathy patients. Exposure level to OTA. Exp. Toxicol. Pathol. 63, 613-618.

Zain, M.E., 2011. Impact of mycotoxins on humans and animals. J. Saudi Chem. Soc. 15, $129-144$

Zhang, H., Dong, M., Yang, Q., Apaliya, M.T., Li, J., Zhang, X., 2016. Biodegradation of zearalenone by Saccharomyces cerevisiae: Possible involvement of ZEN responsive proteins of the yeast. J. Proteome 143, 416-423.

Zhao, L., Jin, H.T., Lan, J., Zhang, R.Y., Ren, H.B., Zhang, X.B., Yu, G.P., 2015. Detoxification of zearalenone by three strains of Lactobacillus plantarum from fermented food in vitro. Food Control 54, 158-164. 